\title{
Análise de Variância Fatorial do Desempenho de Estudantes sob a Influência de Aprimoramentos Progressivos em Plataformas de Apoio ao Ensino
}

\author{
Taffarel Brant-Ribeiro ${ }^{1}$, Renan Gonçalves Cattelan ${ }^{2}$ \\ ${ }^{1}$ Instituto Federal de Educação, Ciência e Tecnologia do Sul de Minas Gerais \\ Passos/MG, Brasil \\ ${ }^{2}$ Faculdade de Computação - Universidade Federal de Uberlândia \\ Uberlândia/MG, Brasil \\ brant.ribeiro@ifsuldeminas.edu.br, renan@ufu.br
}

\begin{abstract}
Usage of new educational technologies still raises questions about the efficiency of these approaches and which benefits they provide to academic field. Several researches that study these technologies are only concerned with user comparisons, not focusing on investigating to what extent the improvement of tools tends to maintain influence on students' performance. Based on this, we developed an Experiments Manager capable of controlling the visibility of new functionalities added to Classroom eXperience $(C X)$ platform and, using it, a Factorial Analysis of Variance was performed to understand if these enhancements brought significant improvements to students' grades. We noticed that students' performance was influenced by the interaction between CX functionalities and taken courses. In all undergraduate classes, there were significant increases on students' performance in a comparison between $C X$ absence and its use with Socialization and Gamification modules. In graduate classes, no significant difference in students' grades were found, although there were also increases on students' performance at this academic level.
\end{abstract}

Resumo. O emprego de novas tecnologias educacionais ainda gera questões acerca da eficiência dessas abordagens e quais benefícios elas propiciam ao âmbito acadêmico. Diversas pesquisas que estudam tais tecnologias preocupam-se apenas em fazer comparações entre usuários, não dedicando-se em investigar até que ponto o aprimoramento das ferramentas tende a manter alguma influência sobre o desempenho dos estudantes. Com base nisso, este trabalho desenvolveu um Gerenciador de Experimentos capaz de controlar a exibição de novas funcionalidades acrescentadas à plataforma Classroom eXperience $(C X)$ e, empregando-o, foi realizada uma Análise de Variância Fatorial para se compreender se esses aprimoramentos trouxeram melhorias significativas nas notas dos alunos. Percebeu-se que o desempenho dos estudantes foi influenciado pela interação entre as funcionalidades do CX e as disciplinas cursadas. Em todas as turmas de graduação, houve aumentos significativos nas notas dos alunos num comparativo entre a ausência do CX e seu uso junto aos módulos de Socialização e Gamificação. Na pós-graduação, não se observou diferença significativa no desempenho discente, embora também tenham havido acréscimos no desempenho obtido pelos estudantes nesse nível acadêmico. 
VII Congresso Brasileiro de Informática na Educação (CBIE 2018)

Anais do XXIX Simpósio Brasileiro de Informática na Educação (SBIE 2018)

\section{Introdução}

O uso de novas propostas tecnológicas no escopo educacional ainda gera questões acerca da eficiência dessas abordagens e quais benefícios elas propiciam de fato ao âmbito acadêmico [Schmidt et al., 2014, Knight et al., 2015, Gasevic et al., 2017, Cai, 2018]. Investigações nessa vertente abrangem uma linha de pesquisa denominada Learning Analytics (LA), compreendida como o processo de se coletar e analisar dados de discentes com o intuito de, por exemplo, antever desempenhos futuros e compreender fluxos de aprendizagem [Barneveld et al., 2012, Chatti et al., 2014, Yassine et al., 2016].

Formalizada inicialmente em 2010, a área de LA é uma temática de pesquisa recente, originada a partir da interseção de diversas vertentes científicas tais como a Psicologia, a Ciência da Computação, a Pedagogia e a Ciência da Aprendizagem [Ferguson, 2012, Chatti et al., 2014, Kortemeyer, 2017]. Pelo seu caráter contemporâneo e multidisciplinar, a LA ainda não dispõe de um arcabouço bem fundamentado de métodos para a realização e replicação de pesquisas científicas. Na literatura, trabalhos sobre o desempenho de usuários são frequentemente observados e abrem margem para diversas análises, tais como a verificação de ganhos para estudantes que fazem uso cotidiano de ferramentas acadêmicas [Settle et al., 2011, Mouri et al., 2015].

No entanto, a maioria destas pesquisas preocupa-se apenas em fazer comparações entre usuários, expondo de maneira repetitiva a existência de situações em que ocorrem aumentos significativos de desempenho entre grupos analisados [Dickson et al., 2012, Mouri et al., 2015]. Até mesmo em trabalhos que fazem o acompanhamento de ferramentas acadêmicas por períodos mais extensos e abordam quantidades maiores de usuários [Settle et al., 2011, Danielson et al., 2014, Asarta e Schmidt, 2017], observa-se a realização de análises semelhantes sobre as mesmas funcionalidades, apresentando mudanças nos indivíduos observados, mas sem a elaboração de novas hipóteses de pesquisa. Percebe-se, portanto, que na literatura os mesmos objetivos de pesquisa tendem a ser empregados de maneira exaustiva, o que causa certa previsibilidade de resultados e a impressão de esgotamento de assunto sobre a temática.

Com base nisso, a presente pesquisa teve como objetivo analisar se o processo de aprimoramento progressivo de plataformas de apoio ao ensino realmente tende a manter elevações significativas no desempenho de discentes que estão em contato com essas ferramentas no cotidiano acadêmico. Para isso, inicialmente foi desenvolvido um Gerenciador de Experimentos capaz de controlar a exibição das funcionalidades de uma plataforma de apoio ao ensino intitulada Classroom eXperience (CX). Empregando este gerenciador, foi realizada uma Análise de Variância Fatorial 4x5 em 501 observações referentes às notas obtidas por estudantes de cursos de graduação em Sistemas de Informação e graduação e pós-graduação em Ciência da Computação no decorrer de seis anos letivos. Tal análise investigou até que ponto o acréscimo de novas funcionalidades no $\mathrm{CX}$ gerou aumentos nas notas dos estudantes que utilizaram essa ferramenta e se de fato algum nível acadêmico (como a graduação ou a pós-graduação) se mostrou mais influenciável por este tipo de abordagem de apoio ao ensino.

Deste modo, o restante deste trabalho está estruturado da seguinte maneira: na Seção 2, o Gerenciador de Experimentos desenvolvido é detalhado; na Seção 3, o processo experimental é demonstrado; na Seção 4, os resultados obtidos são apresentados e discutidos; e, por fim, conclusões e considerações finais são pontuadas na Seção 5. 
VII Congresso Brasileiro de Informática na Educação (CBIE 2018)

Anais do XXIX Simpósio Brasileiro de Informática na Educação (SBIE 2018)

\section{Gerenciador de Experimentos}

Devido ao escopo de investigação desta pesquisa compreender muitas amostras com níveis distintos de tratamentos, viu-se a necessidade de se desenvolver um módulo de gerenciamento capaz de estruturar as etapas de análise sem a ocorrência de desorganizações que levariam à perda de confiabilidade nos resultados obtidos. Com isso em mente, foi desenvolvido e implementado um Gerenciador de Experimentos com características generalistas e capaz de ser empregado para realizar o controle da exibição de módulos e funcionalidades em plataformas de apoio ao ensino, tais como Ambientes Educacionais Ubíquos (AEUs) ou Ambientes Virtuais de Aprendizagem (AVAs).

Este gerenciador compreendeu um mecanismo capaz de realizar o controle de quais funcionalidades deveriam ser exibidas para usuários, de modo a ser possível habilitá-las em tratamentos diferentes e para grupos distintos de indivíduos simultaneamente. Para esta finalidade, considerou-se um módulo de monitoramento capaz de receber dados advindos de sistemas educacionais e empregá-los de modo a criar estruturas de controle para se definir a visibilidade de funcionalidades (Figura 1).

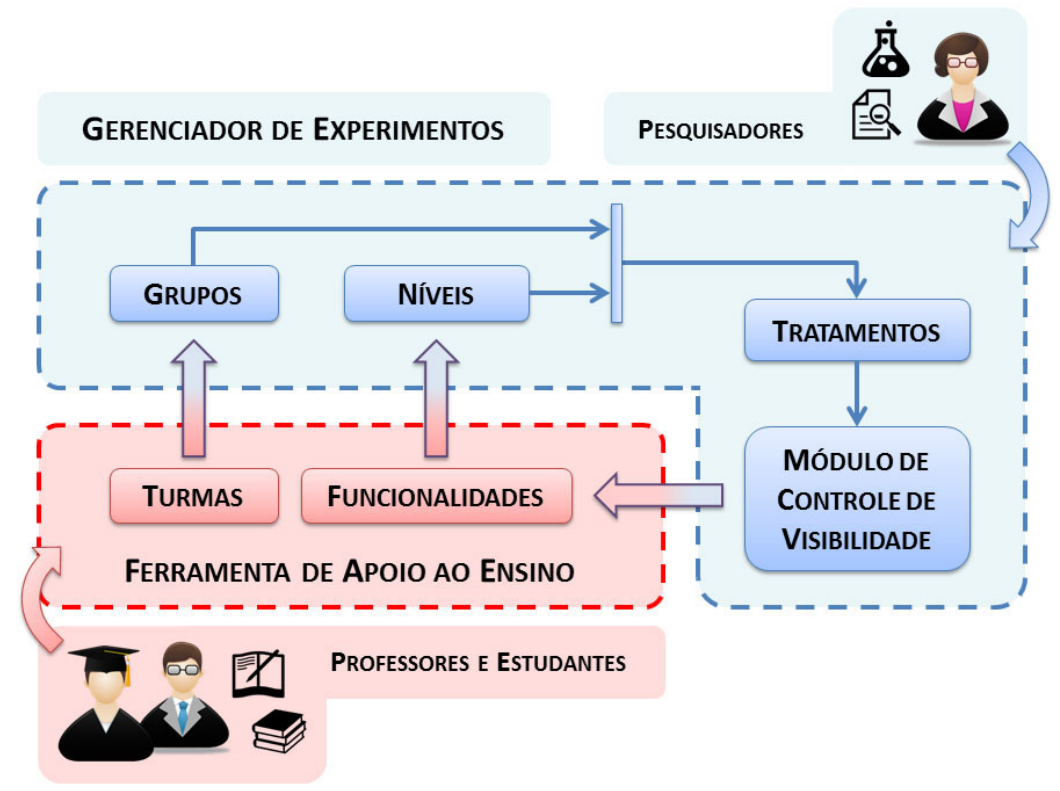

Figura 1. Estrutura do Gerenciador de Experimentos e interface de comunicação com ferramentas de apoio ao ensino (Fonte: Autoria própria).

O Gerenciador de Experimentos (realçado em azul na Figura 1) compreende um módulo de organização de dados provenientes de ferramentas acadêmicas. No âmbito de plataformas educacionais, as turmas que possuem estudantes matriculados em disciplinas cadastradas nessas ferramentas são caracterizadas como grupos (ou amostras) experimentais a serem utilizados pelo gerenciador. Além disso, cada funcionalidade presente na plataforma fornece dados referentes aos níveis de acesso a essas funcionalidades, visto que as mesmas podem ser habilitadas ou desabilitadas para cada usuário ou, também, para conjuntos distintos de usuários que fazem parte de uma mesma turma.

Empregando-se os dados dos grupos e dos níveis de acesso às funcionalidades, os pesquisadores podem montar tratamentos, que são organizações dos níveis de visibilidade das funcionalidades que serão habilitadas para cada grupo de estudantes. Esses 
VII Congresso Brasileiro de Informática na Educação (CBIE 2018)

Anais do XXIX Simpósio Brasileiro de Informática na Educação (SBIE 2018)

tratamentos são organizados utilizando-se um módulo de controle de visibilidade das funcionalidades nas plataformas, capaz de regular e restringir o que cada usuário poderá acessar em cada uma das turmas que fizer parte. Por se tratar de um elemento capaz de realizar o controle de funcionalidades e módulos que compõem as ferramentas de apoio ao ensino, apenas pesquisadores devem ter acesso ao módulo de gerenciamento. Para a presente pesquisa, este Gerenciador de Experimentos foi implementado na plataforma de apoio ao ensino Classroom eXperience (CX), que se trata de uma ferramenta que já possui professores e alunos que a utilizam no decorrer dos semestres letivos.

\subsection{Classroom eXperience $(\mathrm{CX})$}

O CX ${ }^{1}$ [Brant-Ribeiro et al., 2015] é um AEU desenvolvido com o intuito de se registrar fluxos de conteúdo multimídia apresentados em sala de aula e disponibilizá-los posteriormente para alunos e professores. Por meio de seu uso, é possível fazer a captura, armazenamento, acesso e extensão das mídias geradas em ambientes educacionais e contribuir com os processos de ensino e aprendizagem de usuários que utilizam essa tecnologia no cotidiano. Desta maneira, o sistema dispõe de componentes de hardware e software especializados para realizar a gravação das atividades educacionais, sincronizar automaticamente os fluxos de mídia concebidos e disponibilizar esse conteúdo por meio de uma interface de acesso Web, cujas telas podem ser observadas na Figura 2.

(a)

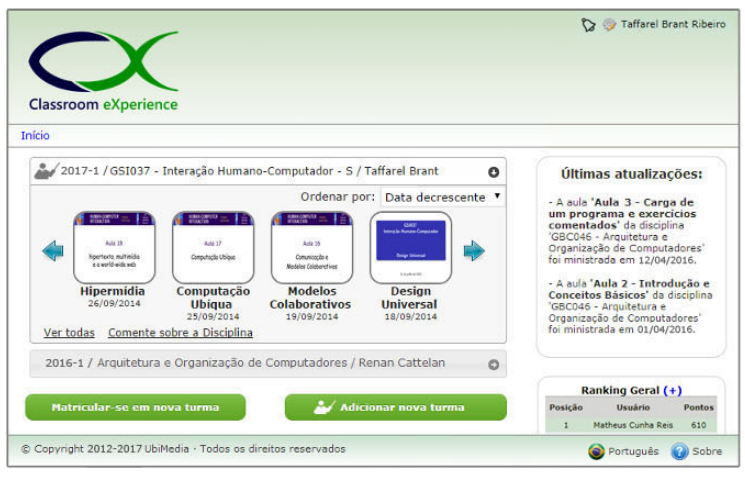

(b)

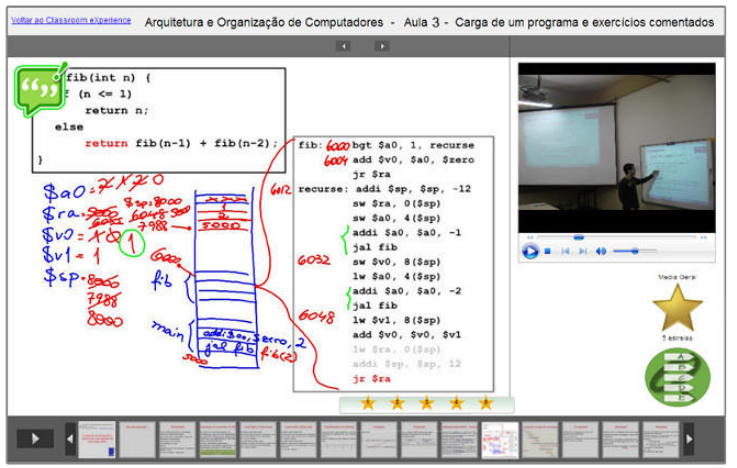

Figura 2. Interface Web do CX: (a) tela principal e (b) tela de aula capturada (Fonte: Adaptado de [Brant-Ribeiro e Cattelan 2015]).

Ao fazerem login no sistema, os usuários são direcionados para a tela principal (Figura 2(a)), na qual é possível, para um professor, adicionar uma nova turma e, para os alunos, matricularem-se nas turmas já cadastradas no sistema. Além disso, esta página exibe as últimas atualizações que aconteceram no $\mathrm{CX}$, tais como aulas capturadas recentemente e avaliações registradas pelos professores - como provas, trabalhos, exercícios e seminários. Ao selecionarem uma aula específica, os usuários devem escolher em qual formato de apresentação o conteúdo capturado será exibido. No CX, atualmente há três tipos de formato de apresentação: somente slides, somente vídeo e completo. Caso selecione a primeira opção, o usuário será direcionado para uma página contendo os slides utilizados pelo professor durante uma sessão de captura, já com as anotações feitas nessas mídias. A segunda opção apresentará apenas o vídeo de uma aula capturada pelo sistema e a terceira exibirá ambos os fluxos de mídia, conforme a Figura 2(b).

\footnotetext{
${ }^{1}$ http://cx.facom.ufu.br/
} 
VII Congresso Brasileiro de Informática na Educação (CBIE 2018)

Anais do XXIX Simpósio Brasileiro de Informática na Educação (SBIE 2018)

Percebe-se também na Figura 2 a existência de funcionalidades que fazem parte do Módulo Social e de Gamificação do CX [Brant-Ribeiro et al., 2015]. Na Figura 2(a), um ranking apresenta o placar das pontuações relativas às atividades realizadas pelos estudantes que utilizam o CX. Na Figura 2(b), é possível observar um botão para a inserção de comentários nos slides, uma área de ranqueamento de slides empregando estrelas, uma região com um feedback da média de estrelas para cada slide e uma opção para responder aos quizzes que foram previamente cadastrados pelos professores.

\section{Experimentos}

Para a realização desta pesquisa, foram utilizados dados provenientes de vinte turmas que cursaram disciplinas da grade curricular dos cursos de graduação em Sistemas de Informação e graduação e pós-graduação em Ciência da Computação da Faculdade de Computação da Universidade Federal de Uberlândia (FACOM/UFU), totalizando 501 observações. A variável analisada foi o desempenho obtido pelos estudantes em disciplinas cursadas durante seis anos letivos (de 2012 a 2017) e, para isso, as notas alcançadas pelos discentes foram coletadas ao final de cada semestre.

Embora o tamanho das amostras utilizadas para a presente pesquisa não tenha se mantido fixo, respeitou-se a estimativa de tamanho ótimo amostral previamente determinada em [Brant-Ribeiro e Cattelan 2015] e assegurou-se que as amostras cumprissem um mínimo médio de 25 estudantes por turma - o que garantiu a confiabilidade nos resultados obtidos neste trabalho. Deste modo, inicialmente as observações coletadas (notas dos estudantes) foram submetidas ao teste de Shapiro-Wilk para verificar se os resíduos da variável observada seguiam a distribuição normal e ao teste de Levene para averiguar se as amostras possuíam variâncias homogêneas [Triola, 2014].

Posteriormente, realizou-se uma Análise de Variância Fatorial 4x5 (Two-way ANOVA) para se atestar a existência de diferenças significativas entre os níveis de dois fatores investigados. O primeiro fator, denominado "Uso do CX", foi caracterizado como o modo com que o CX foi empregado pelos estudantes e possuiu quatro níveis distintos: 1) $\mathrm{CX}$ Ausente, 2) Uso isolado do CX, 3) Uso do CX + Módulo Social (MS) e 4) Uso do CX + MS + Gamificação. O segundo fator, intitulado "Disciplinas", compreendeu todas as disciplinas que foram cursadas pelos alunos empregando os níveis de uso do CX supracitados e possuiu cinco níveis: Lógica Matemática (LM), Otimização, Arquitetura e Organização de Computadores (AOC) e Interação Humano-Computador (IHC) (nos níveis de graduação e pós-graduação).

Para a realização da ANOVA, inicialmente foi executado o teste $F$ de Snedecor com o intuito de se atestar a existência de diferenças significativas entre as médias dos níveis dos fatores analisados [Snedecor e Cochran, 1980]. Este teste também foi utilizado para se observar a existência de uma interação entre a utilização do CX e as disciplinas cursadas pelos estudantes, de modo a ser possível compreender se o desempenho obtido pelos alunos mostrava-se influenciável tanto pelo tipo da disciplina cursada quanto pelo nível de uso do $\mathrm{CX}$ durante os semestres letivos. Como o teste $F$ de Snedecor não se mostra capaz de indicar especificamente quais são os níveis que possuem as médias significativamente diferentes, por fim empregou-se o teste de Tukey para realizar a comparação de todas as médias amostrais executando análises consecutivas entre os pares de amostras até que todas as possibilidades combinatórias fossem realizadas [Triola, 2014]. 
VII Congresso Brasileiro de Informática na Educação (CBIE 2018)

Anais do XXIX Simpósio Brasileiro de Informática na Educação (SBIE 2018)

\section{Resultados e Discussões}

Os testes de Shapiro-Wilk, Levene e $F$ de Snedecor possibilitaram atestar a existência de diferenças entre os níveis dos fatores "Uso do CX" e "Disciplinas". Como resultados, na Tabela 1 é possível observar que a ANOVA do desempenho de estudantes apresentou efeito significativo em três circunstâncias distintas: para o uso do CX (F1), para as disciplinas cursadas (F2) e também para a interação entre os dois fatores (F1 x F2).

Tabela 1. Análise de Variância Fatorial do desempenho discente com relação ao uso do CX em disciplinas de graduação e pós-graduação em cursos da área computacional (Fonte: Autoria própria).

\begin{tabular}{cccc}
\hline Fatores de Variação $^{1}$ & Graus de Liberdade & Quadrado Médio & $F(P)$ \\
\hline Uso do CX (F1) & 3 & 3263,554 & $24,044(<0,01)$ \\
Disciplinas (F2) & 4 & 3971,876 & $29,263(<0,01)$ \\
Interação (F1 x F2) & 12 & 648,836 & $4,780(<0,01)$ \\
Resíduo & 481 & 135,730 & \\
\hline$C V(\%) ; W(P) ; F^{\prime}(P)$ & 14,$92 ; 0,0961(<0,01) ; 3,110(0,062)$ \\
\hline
\end{tabular}

${ }^{1} F(P)$ : Estatística e Probabilidade do teste $F$ de Snedecor; Probabilidades menores que 0,05 indicam a existência de ao menos um tratamento (nível) diferente dos demais; $C V$ : Coeficiente de Variação; $W(P)$ e $F^{\prime}(P)$ : Estatísticas dos testes de Shapiro-Wilk e Levene; Probabilidades maiores que 0,05 indicam resíduos com distribuição normal e variâncias homogêneas, respectivamente.

Em razão disso, o maior impacto deste resultado incidiu sobre a característica de interação entre o uso do CX e as disciplinas cursadas pelos estudantes, ou seja, o desempenho dos alunos mostrou-se de fato influenciado tanto pelo nível em que a ferramenta foi utilizada quando pelo tipo de disciplina cursada. Além disso, também é possível observar que o Coeficiente de Variação (CV) experimental alcançado mostrou-se baixo $(14,92 \%)$, garantindo uma variabilidade sintetizada para esta pesquisa.

Por ter havido um efeito significativo de interação entre os fatores "Uso do CX" e "Disciplinas" (Tabela 1), também realizaram-se os desdobramentos deste resultado comparando-se os pares das médias amostrais do desempenho dos estudantes pelo teste de Tukey (Tabelas 2 e 3).

Ao se analisar o desdobramento da interação com enfoque nas disciplinas cursadas pelos estudantes (Tabela 2), é possível perceber que o uso do CX resultou em acréscimos gradativos no desempenho dos alunos. As disciplinas LM e IHC (Graduação) apresentaram resultados equivalentes, alcançando aumentos significativos nas médias de desempenho logo após a inclusão do módulo de Gamificação (nível D) no CX. No entanto, as turmas dessas disciplinas que empregaram o CX isoladamente (nível B) ou com apenas a inclusão do MS (nível C) não apresentaram acréscimos em suas notas, mostrando-se similares às turmas que cursaram tais disciplinas sem a utilização do CX (nível A).

Na disciplina Otimização, a turma que empregou o CX isoladamente (nível B) não apresentou um desempenho superior ao daquela que não o utilizou, fato similar ao ocorrido em LM e IHC (Graduação). No entanto, em Otimização o acréscimo do MS (nível C) provocou um aumento significativo das notas, gerando uma elevação no desempenho 
VII Congresso Brasileiro de Informática na Educação (CBIE 2018)

Anais do XXIX Simpósio Brasileiro de Informática na Educação (SBIE 2018)

Tabela 2. Desempenho discente com relação ao uso do CX em disciplinas de graduação e pós-graduação em cursos da área computacional com enfoque nas disciplinas cursadas pelos estudantes (Fonte: Autoria própria).

\begin{tabular}{ccccccc}
\hline \multirow{2}{*}{ Uso do CX } & \multicolumn{5}{c}{ Disciplinas } & \multirow{2}{*}{ Média } \\
\cline { 2 - 6 } & LM & Otimização & AOC & IHC (Grad) & IHC (Pós) & \\
\hline A & $72,28 B$ & $61,86 B$ & $62,62 B$ & $77,83 B$ & $84,94 A$ & 71,11 \\
B & $78,74 A B$ & $62,25 B$ & $75,34 A$ & $83,94 A B$ & $90,94 A$ & 77,88 \\
C & $79,29 A B$ & $79,20 A$ & $80,19 A$ & $80,70 A B$ & $87,54 A$ & 80,77 \\
D & $84,27 A$ & $82,15 A$ & $75,42 A$ & $85,45 A$ & $95,64 A$ & 82,76 \\
\hline Média & 78,88 & 71,73 & 73,40 & 81,88 & 89,28 & \\
\hline
\end{tabular}

${ }^{1}$ Médias seguidas por letras distintas maiúsculas nas colunas diferem-se entre si pelo teste de Tukey a 0,05 de significância; LM: Lógica Matemática; AOC: Arquitetura e Organização de Computadores; IHC: Interação Humano-Computador; Grad: Graduação; Pós: Pós-graduação; A: CX Ausente; B: Uso isolado do CX; C: Uso do CX junto ao Módulo Social; D: Uso do CX junto aos Módulos Social e de Gamificação.

tanto dos alunos que utilizaram o módulo social isoladamente quanto daqueles que o empregaram com a Gamificação. Ainda neste escopo, a partir da inserção do CX isolado na disciplina AOC (nível B), já foi possível observar um aumento do desempenho dos alunos e essa característica se manteve em todos os demais níveis de uso do CX.

Em suma, para todas as turmas de graduação analisadas nesta pesquisa, ocorreram aumentos significativos no desempenho dos alunos num comparativo direto entre a ausência do CX (nível A) e a utilização desta ferramenta junto aos módulos de Socialização e Gamificação (nível D). Analisando as disciplinas de Otimização e AOC, é possível perceber que este aumento ocorreu ainda antes, pois em Otimização a inclusão do MS já gerou aumentos robustos e em AOC a inserção isolada do CX já garantiu a elevação do desempenho acadêmico discente.

Para IHC (Pós-graduação), não se observou diferença no desempenho dos alunos entre quaisquer níveis de uso do CX. Pressupõe-se que, conforme indicado por [Brant-Ribeiro et al., 2015], alunos de pós-graduação normalmente já possuem seus próprios métodos de estudo e tendem a ser esforçados - algo que faz com que seus níveis de desempenho sejam naturalmente elevados com ou sem a utilização de tecnologias. A inserção de novas tecnologias neste escopo acaba se configurando como mais uma opção, diluindo sua importância e conveniência de uso do sistema.

Tal característica pode ser confirmada ao se analisar o desdobramento da ANOVA com enfoque nos níveis de utilização do CX (Tabela 3), pois a disciplina IHC (Pósgraduação) se manteve com os maiores desempenhos em todas as circunstâncias observadas. Acerca das turmas que não empregaram o CX durante os semestres letivos (nível A), o desempenho das disciplinas de IHC (tanto na graduação quanto na pós-graduação) mostrou-se similar, ao passo que Otimização e AOC apresentaram desempenhos menores e LM obteve um resultado intermediário e superior a essas, mas que não se diferiu significativamente de IHC (Graduação). 
VII Congresso Brasileiro de Informática na Educação (CBIE 2018)

Anais do XXIX Simpósio Brasileiro de Informática na Educação (SBIE 2018)

Tabela 3. Desempenho discente com relação ao uso do CX em disciplinas de graduação e pós-graduação em cursos da área computacional com enfoque nos níveis de utilização do CX (Fonte: Autoria própria).

\begin{tabular}{|c|c|c|c|c|c|c|}
\hline \multirow{2}{*}{ Uso do $\mathrm{CX}^{1}$} & \multicolumn{5}{|c|}{ Disciplinas } & \multirow{2}{*}{ Média } \\
\hline & LM & Otimização & $\mathrm{AOC}$ & IHC (Grad) & IHC (Pós) & \\
\hline A & $72,28 b$ & $61,86 c$ & $62,62 c$ & $77,83 a b$ & $84,94 a$ & 71,11 \\
\hline B & $78,74 b$ & $62,25 c$ & $75,34 b$ & $83,94 a b$ & $90,94 a$ & 77,88 \\
\hline $\mathrm{C}$ & $79,29 a$ & $79,20 a$ & $80,19 a$ & $80,70 a$ & $87,54 a$ & 80,77 \\
\hline $\mathrm{D}$ & $84,27 b c$ & $82,15 b c$ & $75,42 c$ & $85,45 b$ & $95,64 a$ & 82,76 \\
\hline Média & 78,88 & 71,73 & 73,40 & 81,88 & 89,28 & \\
\hline
\end{tabular}

${ }^{1}$ Médias seguidas por letras distintas minúsculas nas linhas diferem-se entre si pelo teste de Tukey a 0,05 de significância; LM: Lógica Matemática; AOC: Arquitetura e Organização de Computadores; IHC: Interação Humano-Computador; Grad: Graduação; Pós: Pós-graduação; A: CX Ausente; B: Uso isolado do CX; C: Uso do CX junto ao Módulo Social; D: Uso do CX junto aos Módulos Social e de Gamificação.

Para o uso isolado do CX (nível B), as disciplinas de IHC mantiveram-se semelhantes. AOC e LM apresentaram desempenhos similares aos de IHC (Graduação), enquanto Otimização manteve-se com o menor desempenho dentre as disciplinas nesta circunstância. Com a inserção do MS (nível C), todas as disciplinas mostraram-se semelhantes, ao passo que com a inclusão da Gamificação (nível D), a pós-graduação destacou-se novamente com um desempenho maior que os demais.

Analisando-se apenas as disciplinas de graduação no último nível de uso do CX, é possível verificar que AOC apresentou o menor desempenho dentre as disciplinas. De todo modo, este não deve ser considerado um desempenho ruim, devido à disciplina ter apresentado uma média de 75,42. Já as demais disciplinas alcançaram desempenhos superiores, ainda que LM e Otimização não tenham se diferenciado significativamente de AOC. A disciplina IHC (Graduação) obteve o melhor resultado entre as disciplinas de graduação com o uso da Gamificação, alcançando um desempenho médio de 85,45.

Por fim, é possível observar que todas as disciplinas apresentaram acréscimos gradativos nos desempenhos obtidos nesta pesquisa. A disciplina de IHC (Pós-graduação) manteve-se acima das demais para todos os níveis de uso do CX, ao passo que IHC (Graduação) e LM mantiveram-se intermediárias e permaneceram próximas entre si. Otimização e AOC também tiveram aumentos consideráveis de desempenho e tenderam a se aproximar das demais disciplinas a partir das inserções dos módulos de Socialização e Gamificação no CX.

\section{Conclusões}

Esta pesquisa teve como objetivo realizar uma ANOVA Fatorial $4 \times 5$ para se atestar a existência de diferenças significativas entre os fatores "Uso do CX" e "Disciplinas" sobre o desempenho acadêmico de estudantes em cursos de graduação em Sistemas de Informação e graduação e pós-graduação em Ciência da Computação. Para isso, inicialmente foi projetado e desenvolvido um Gerenciador de Experimentos com o intuito de 
VII Congresso Brasileiro de Informática na Educação (CBIE 2018)

Anais do XXIX Simpósio Brasileiro de Informática na Educação (SBIE 2018)

se organizar o nível de visibilidade das funcionalidades presentes em ferramentas de apoio ao ensino e se automatizar o planejamento experimental de pesquisas.

Em seguida, realizou-se uma ANOVA que empregou 501 observações referentes aos desempenhos obtidos pelos estudantes ao final dos semestres letivos. A partir desta análise, percebeu-se preliminarmente a existência de efeitos significativos em três casos: na influência do uso do CX sobre as notas dos estudantes, no efeito que disciplinas diferentes cursadas pelos alunos causaram em seus desempenhos e, também, na interação existente entre estes dois fatores. Em razão disso, o maior impacto deste resultado refletiu-se diretamente sobre as características de interação entre a utilização do CX e as disciplinas cursadas pelos estudantes, ou seja, o desempenho dos alunos pode ser compreendido como uma variável influenciada tanto pelas funcionalidades da ferramenta quanto pelo tipo de disciplina cursada pelos estudantes, ambos em conjunto.

Para todas as turmas de graduação investigadas neste trabalho, houve aumentos significativos no desempenho dos estudantes num comparativo direto entre a ausência do CX e a utilização desta ferramenta junto aos módulos de Socialização e Gamificação. Numa análise específica acerca das disciplinas de Otimização e AOC, foi possível concluir que este aumento ocorreu ainda antes, pois em Otimização a inclusão do MS já foi capaz de gerar aumentos significativos nas notas e em AOC a inserção isolada do CX já garantiu um aumento robusto no desempenho discente.

No entanto, para a disciplina de IHC (Pós-graduação) não se observou diferença significativa no desempenho dos alunos entre quaisquer níveis de uso do $\mathrm{CX}$. Isso permite deduzir que estudantes de pós-graduação já possuem seus próprios hábitos e métodos de estudo, conseguindo obter notas naturalmente mais elevadas que os alunos de graduação. É possível concluir, portanto, que propostas de novas tecnologias educacionais para pósgraduandos acabam se mostrando como mais uma opção elencável, o que dilui a importância e conveniência de utilização da plataforma para este público.

Além disso, durante a realização desta pesquisa também foi observado um fator limitante referente às disciplinas que foram avaliadas empregando-se a ANOVA. Das cinco disciplinas cursadas pelas vinte turmas utilizadas nesta análise, apenas uma delas foi cursada por estudantes de pós-graduação. Os resultados obtidos e as discussões feitas com os dados das turmas de pós-graduação refletem, portanto, o comportamento que os alunos tiveram especificamente na disciplina de IHC. Enxerga-se, portanto, a necessidade de se realizar uma ANOVA englobando mais turmas com estudantes pós-graduandos, de modo a ser possível analisar adequadamente o impacto que ferramentas de apoio ao ensino causam no desempenho de turmas de pós-graduação em ambientes acadêmicos reais.

\section{Agradecimentos}

O presente trabalho foi realizado com apoio da Coordenação de Aperfeiçoamento de Pessoal de Nível Superior - Brasil (CAPES) - Código de Financiamento 001. Os autores também agradecem às agências de pesquisa FAPEMIG e CNPq, ao PET/SESu/MEC e ao Instituto Federal do Sul de Minas Gerais (IFSULDEMINAS) pelo apoio concedido.

\section{Referências}

Asarta, C. J. e Schmidt, J. R. (2017). Comparing student performance in blended and traditional courses: Does prior academic achievement matter? The Internet and Higher 
VII Congresso Brasileiro de Informática na Educação (CBIE 2018)

Anais do XXIX Simpósio Brasileiro de Informática na Educação (SBIE 2018)

Education, 32:29-38.

Barneveld, A. V., Arnold, K. E. e Campbell, J. P. (2012). Analytics in Higher Education: Establishing a Common Language. EDUCAUSE Learning Initiative, 1:1-11.

Brant-Ribeiro, T., Biase, N. G. e Cattelan, R. G. (2015). Impacto de Aplicações de Captura e Acesso em Estudantes de Cursos de Computação. RBIE, 23(2):111-126.

Brant-Ribeiro, T. e Cattelan, R. (2015). Tamanho Ótimo de Amostra para Análise do Desempenho de Estudantes em Ambientes Educacionais Ubíquos. In Anais do SBIE, volume 26, pages 31-40, Maceió, BR. SBIE.

Cai, S. (2018). Case studies of augmented reality applications for authentic learning. In Authentic Learning Through Advances in Technologies, p. 115-134. Springer.

Chatti, M. A., Lukarov, V., Thüs, H., Muslim, A., Yousef, A. M. F., Wahid, U., Greven, C., Chakrabarti, A. e Schroeder, U. (2014). Learning Analytics: Challenges and Future Research Directions. ELEED, 10(1).

Danielson, J., Preast, V., Bender, H. e Hassall, L. (2014). Is the Effectiveness of Lecture Capture Related to Teaching Approach or Content Type? C\&E, 72:121-131.

Dickson, P. E., Warshow, D. I., Goebel, A. C., Roache, C. C. e Adrion, W. R. (2012). Student Reactions to Classroom Lecture Capture. In Proc. of the $17^{\text {th }}$ ACM ITiCSE, p. 144-149.

Ferguson, R. (2012). Learning Analytics: Drivers, Developments and Challenges. IJTEL, 4(5):304-317.

Gasevic, D., Mirriahi, N., Dawson, S. e Joksimovic, S. (2017). Effects of instructional conditions and experience on the adoption of a learning tool. Comput Human Behav, 67:207-220.

Knight, D. B., Brozina, C., Stauffer, E. M., Frisina, C. e Abel, T. D. (2015). Developing a learning analytics dashboard for undergraduate engineering using participatory design. In 2015 ASEE Annual Conference \& Exposition.

Kortemeyer, G. (2017). The Spectrum of Learning Analytics. ELEED, 12(1).

Mouri, K., Ogata, H. e Uosaki, N. (2015). Analysis of ubiquitous learning logs using social network analysis. IJMLO, 9(2):101-123.

Schmidt, A., Pfleging, B., Holz, C. e Holmquist, L. E. (2014). From photography to ubiquitous capture systems. Pervasive Computing, IEEE, 13(1):10-13.

Settle, A., Dettori, L. e Davidson, M. J. (2011). Does Lecture Capture Make a Difference for Students in Traditional Classrooms. In Proc. of the $16^{\text {th }}$ ACM ITiCSE, p. 78-82.

Snedecor, G. W. e Cochran, W. G. (1980). Statistical Methods. Iowa State University Press, $7^{\mathrm{a}}$ edição.

Triola, M. F. (2014). Introdução à Estatística - Atualização da Tecnologia. LTC, São Paulo, $11^{\text {a }}$ edição.

Yassine, S., Kadry, S. e Sicilia, M. A. (2016). Learning Analytics and Learning Objects Repositories: Overview and Future Directions. Learning, Design, and Technology, p. 1-29. Springer. 